\title{
A Structure and Durability Comparison of Membrane Electrode Assembly Fabrication Methods: Self-Assembled Versus Hot-Pressed
}

\author{
Jennifer Hack, ${ }^{1,2}$ T. M. M. Heenan, ${ }^{1, *}$ F. Iacoviello, ${ }^{1}$ N. Mansor, ${ }^{1}{ }^{Q}$. Meyer, ${ }^{1}$ P. Shearing, ${ }^{1}$ \\ N. Brandon, ${ }^{2}$ and D. J. L. Brett ${ }^{1, z}$ \\ ${ }^{1}$ Electrochemical Innovation Lab, Department of Chemical Engineering, University College London, London WC1E \\ 7JE, United Kingdom \\ ${ }^{2}$ Department of Earth Sciences and Engineering, Royal School of Mines, Imperial College London, London SWX WYZ, \\ United Kingdom
}

\begin{abstract}
The most common means of fabricating membrane electrode assemblies (MEAs) for polymer electrolyte fuel cells (PEFCs) involves a hot-press step. The conditions used to perform the hot-press impacts the performance and durability of the fuel cell. However, the hot-press process is not essential for achieving operational MEAs and some practitioners dispense with the hot-press stage altogether by using a self-assembled approach. By performing the integration of the components in-situ during fuel cell assembly, there is the potential to lower the cost and time of manufacture. This study investigates the electrochemical performance and mechanical microstructure of MEAs that were either hot-pressed or self-assembled (non-hot-pressed) and compared at beginning-of-test (BOT) and end-of-test (EOT), following accelerated stress testing. Hot-pressed and self-assembled MEAs were found to show negligible difference in their performance and almost identical performance degradation. X-ray computed tomography (X-ray CT) showed distinct differences in the microstructure of the electrodes. In addition to a crack network in the catalyst layer, the self-assembled samples exhibit indentations that were not present in the hot-pressed sample. It was concluded that in-situ assembly of MEAs could be a suitable means of fabricating PEFC MEAs.

(C) The Author(s) 2018. Published by ECS. This is an open access article distributed under the terms of the Creative Commons Attribution 4.0 License (CC BY, http://creativecommons.org/licenses/by/4.0/), which permits unrestricted reuse of the work in any medium, provided the original work is properly cited. [DOI: 10.1149/2.0051806jes]

(cc) BY
\end{abstract}

Manuscript submitted December 22, 2017; revised manuscript received February 6, 2018. Published February 21, 2018. This paper is part of the JES Focus Issue on Proton Exchange Membrane Fuel Cell (PEMFC) Durability.

Fuel cells are a promising alternative energy conversion technology, ${ }^{1}$ with polymer electrolyte fuel cells (PEFCs) being particularly suitable for transport applications. ${ }^{2}$ Their low operating temperatures of between typ. $50-100^{\circ} \mathrm{C}$, along with their high electrical efficiencies, make them suitable for replacement of the internal combustion engine. ${ }^{2}$

However, issues surrounding degradation and cost remain a challenge for the improvement of PEFCs. Most of these challenges stem from the materials and manufacture of the membrane electrode assembly (MEA) that consists of two electrodes, the anode and cathode, which are separated by a polymer electrolytic membrane, most commonly Nafion. The electrodes themselves comprise of several layers that have been designed to provide the best electronic conductivity and gas diffusivity, whilst maintaining the ability for good water and thermal management. ${ }^{3,4}$ The gas diffusion layer (GDL) is typically made up of carbon fibers that have been coated with hydrophobic polytetrafluoroethylene (PTFE) to aid with water management. ${ }^{5}$ Coated onto the GDL is a micro-porous layer (MPL) of carbon nanoparticles, which serves as a 'bridge' between the large pores of the GDL and the nanoscale pores in the catalyst layer (CL). ${ }^{6}$ The CL, which is in direct contact with the Nafion electrolyte in the MEA, is most commonly made of platinum nanoparticles on a carbon support. ${ }^{5,7,8}$

A variety of methods exist for the preparation of the MEA, which broadly follow two main routes, as extensively reviewed by Mehta and Cooper. ${ }^{9}$ One route is the direct coating of the CL, usually prepared as an ink, onto the Nafion membrane to produce a catalyst coated membrane (CCM), with the GDL/MPL then placed directly onto the CL. ${ }^{10}$ Whilst this method is thought to have advantages for improving the contact and ion conductivity between the CL and the membrane, ${ }^{11}$ poor contact between the CL and the MPL has been shown to decrease the performance of the PEFC. ${ }^{12,13}$ Alternatively, MEAs can be prepared by the hot-pressing technique, which was first reported by

*Electrochemical Society Student Member.

${ }^{\mathrm{z} E-m a i l: d . b r e t t @ u c l . a c . u k}$
Ticianelli in $1988 .{ }^{14}$ Electrodes are prepared by coating the CL onto the MPL, which is done most commonly by spraying of a catalyst ink, ${ }^{15}$ screen printing ${ }^{16}$ or electro-depositing the CL onto the MPL surface. ${ }^{17}$ Work by Millington et al. has also highlighted the importance of the solvent on the coating of the CL onto the MEA, ${ }^{18,19}$ since intrinsic properties of the solvent can influence the surface morphology of the resulting electrode. For example, cracks of varying diameter and length can form in the CL surface as a result of the drying process of the solvent, which could affect fuel cell performance due to poor contact between the CL and the Nafion. ${ }^{19}$ The prepared electrodes are then laminated onto the Nafion electrolyte by hot-pressing between two heated plates (platens) at a specified temperature and pressure for a given length of time. ${ }^{16,20-23}$ Studies have been conducted into the effects of various temperatures, durations and pressures that can be used for hot pressing, ${ }^{21,22,24-27}$ including work by the authors, who have explored the optimum temperature for hot pressing, based on electrochemical performance and microstructure..$^{20}$

However, it is known that the formal hot-pressing process is not required for an MEA (made from the same materials as used in the hot-pressed case) to still function effectively; ${ }^{21}$ the MEA is laminated in-situ during fuel cell assembly and operation. While certain practitioners routinely employ the self-assembly route with good performance results, ${ }^{21,22}$ questions arise as to whether the same durability can be achieved with a self-assembled MEA, as well as how the structure differs between the two cases. To assess this, a combination of durability testing and 3D structural characterization has been performed.

In order to simulate fuel cell degradation processes on a timescale that can be carried out in a research laboratory, accelerated stress tests (ASTs) are routinely performed. They are designed to replicate the degradation that would occur in a PEFC over thousands of hours under real-world operation in only tens or hundreds of hours. ${ }^{28}$ Electrochemical characterization of PEFCs in the form of polarization curves and cyclic voltammetry $(\mathrm{CV})$ provide a rigorous understanding of the subtle details in the performance of the fuel cell. Furthermore, when carrying out ASTs that target a particular failure mode, electrochemical 


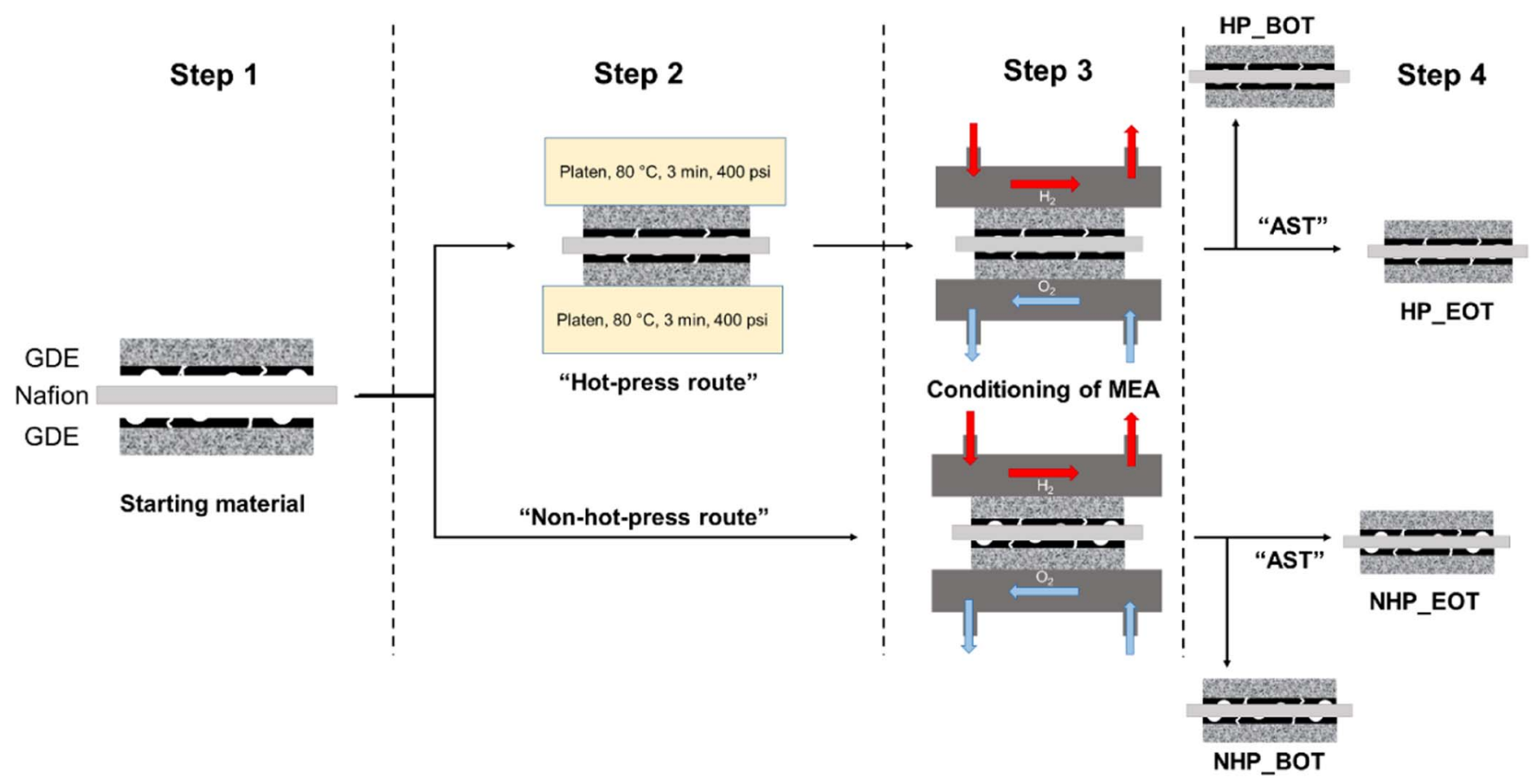

Figure 1. Schematic of the experimental procedure, showing Step 1: starting materials with dents and crack in the GDE, Step 2: hot-pressing or non-hot-pressing step, Step 3: indentations are no longer present in the HP route, but can be seen in the NHP route and Step 4: stage at which X-ray CT was carried out on each sample, with HP_BOT and NHP_BOT after conditioning and HP_EOT and NHP_EOT after AST.

measurements give insight into the mechanisms responsible for degradation. ${ }^{29,30}$ When coupled with imaging techniques, such as $3 \mathrm{D}$ $\mathrm{X}$-ray computed tomography (X-ray CT) and '2D' scanning electron microscopy (SEM), the mechanical evolution of material structure can be correlated to the electrochemical degradation processes and, ultimately, cell performance. ${ }^{20,31,32}$

There are limitations to 2D techniques, such as SEM, in that only surface information can be gained from imaging, which reduces the statistical relevancy of the metrics quantified. In addition, to probe the internal microstructure, the sample must be sectioned for post-mortem analysis, resulting in the destruction of the original MEA structure. ${ }^{25}$ $\mathrm{X}$-ray CT is being increasingly used for PEFC characterization, with the distinct advantage over surface techniques that non-destructive imaging of the internal structures can be carried out. ${ }^{20,33-35}$

Given the state-of-the-art instruments that are now available for 3D characterization, as well as the fact that there is limited discussion of whether the hot pressing procedure is even necessary for MEA preparation, ${ }^{21}$ this paper presents the first in-depth study of hotpressed MEAs compared with self-assembled MEAs. This is done by characterising the cell performance and correlating this to the MEA microstructure. By carrying out AST cycling on the hot-pressed and self-assembled MEAs, the electrochemical performance of the different MEAs can be compared. This gives insight into the durability of the hot-pressed and self-assembled MEAs, by emulating extended cell operation. Finally, X-ray CT was used to probe the internal microstructure of the MEA with sub-micron resolution, which gives an appreciation of the 3D structural properties of the samples. A particular focus on the CL also gives novel insight into the effects of the MEA preparation technique on the CL structure.

\section{Experimental}

Experimental process flow.-A general schematic of the experimental process flow is given in Figure 1, with the experimental procedure being broken down into four main steps. In Step 1, the fresh materials are prepared as two GDEs (for the anode and cathode), and the Nafion electrolyte. Steps 2-4 proceed by two routes, depending on whether the MEA is hot-pressed (HP) or not-hot-pressed (NHP) (this nomenclature is used to refer to all self-assembled MEAs, throughout). The hot pressed route includes the hot-pressing of the MEA in Step 2, whereas the self-assembled route proceeds directly to Step 3. In Step 3, the MEAs are conditioned to prepare two MEAs, namely HP_BOT and NHP_BOT. Steps 1-3 were repeated for two more MEAs, with Step 4 being taken to completion, resulting in two degraded MEAs, namely HP_EOT and NHP_EOT.

Materials.-MEAs consisting of two gas diffusion electrodes (GDEs) separated by an ionomer membrane, were prepared in-house using commercially available materials. GDEs (ELE0162, Johnson Matthey, UK), with a platinum catalyst loading of $0.4 \mathrm{mg} \mathrm{cm}^{-2}$ on each electrode, were cut to an area of $5.29 \mathrm{~cm}^{2}$. Nafion NRE212 membrane (DuPont, USA) was used without pre-treatment. Hotpressed MEAs were prepared by hot-pressing (Carver 4122CE, USA) at $130^{\circ} \mathrm{C}$ for 3 minutes with a pressure of 400 psi. These hot pressing conditions were chosen based on previous work into different hot-pressing temperatures. ${ }^{20}$ NHP MEAs were assembled directly in the fuel cell test housing (Fuel Cell Technologies Inc., USA). MEAs were placed into the fuel cell test housing and closed with a torque of $2.0 \mathrm{~N} \mathrm{~m}$. Four MEA states were investigated: hot-pressed beginningof-test (HP_BOT), non-hot-pressed beginning-of-test (NHP_BOT), hot-pressed end-of-test (HP_EOT) and non-hot-pressed end-of-test (NHP_EOT) (Table I).

Electrochemical characterization.-Electrochemical testing was carried out using a Scribner Associates 850e test rig (Scribner, USA), with inbuilt Scribner 885 potentiostat to control gas-flow, temperature and load parameters. The MEAs were operated at $80^{\circ} \mathrm{C}$, with gases being supplied in flow-through mode at $98 \%$ relative humidity. Stoichiometry of $\mathrm{H}_{2}$ and air was 2 and 6 at the anode and cathode, respectively. MEAs were conditioned by a constant current hold for 30 minutes, at increasing current densities of 50, 100, 300, 500 and $700 \mathrm{~mA}$ $\mathrm{cm}^{-2}$. In accordance with DoE testing recommendations MEAs were considered conditioned if a deviation of less than $5 \mathrm{mV}$ was observed between three polarization curves collected post-conditioning. ${ }^{36}$

Polarization curves were collected between 0 and $1000 \mathrm{~mA} \mathrm{~cm}^{-2}$ at increments of $19 \mathrm{~mA} \mathrm{~cm}^{-2}$ with a hold of $60 \mathrm{~s}$ at each point. 
Table I. List of the four MEAs, their hot-pressing conditions and relevant AST test procedures.

\begin{tabular}{lcc} 
MEA name & $\begin{array}{c}\text { Hot-pressing } \\
\text { Conditions }\end{array}$ & $\begin{array}{c}\text { Accelerated Stress Test } \\
\text { Procedure }\end{array}$ \\
\hline HP_BOT & $\begin{array}{c}3 \text { mins at } 130^{\circ} \mathrm{C} \\
\text { and } 400 \mathrm{psi}\end{array}$ & No accelerated stress tests \\
NHP_BOT & Not hot-pressed & No accelerated stress tests \\
HP_EOT & 3 mins at $130^{\circ} \mathrm{C}$ & 16,000 square-wave \\
& and $400 \mathrm{psi}$ & cycles $0.6 \mathrm{~V}-0.95 \mathrm{~V}$ \\
NHP_EOT & Not hot-pressed & 16,000 square-wave \\
& & cycles $0.6 \mathrm{~V}-0.95 \mathrm{~V}$
\end{tabular}

Three repetitions were measured to confirm a deviation of less than $5 \mathrm{mV}$ between curves. CVs were carried out by flowing nitrogen over the cathode until the OCV was $<0.2 \mathrm{~V}$ and measurements were then collected by sweeping the voltage between $0.05 \mathrm{~V}$ and $1 \mathrm{~V}$ at a scan rate of $50 \mathrm{mV} \mathrm{s}^{-1}$. Electrochemical surface area (ECSA) was calculated from the cyclic voltammogram, by integration of the hydrogen desorption peak of the voltammogram. Further information on the calculation behind this method can be found elsewhere. ${ }^{37}$

The AST procedure was based on the recommendation outlined by the DoE for electrocatalyst-specific degradation ${ }^{38}$ and consisted of 16,000 square-wave cycles between $0.6 \mathrm{~V}$ and $0.95 \mathrm{~V}$, with nitrogen flowing over the cathode. Although the DoE target states a $40 \%$ loss after 30,000 cycles, the experimental results showed over $80 \%$ loss of ECSA after 16,000 cycles, thus the test was considered complete and terminated. An equivalent to 27 hours of voltage cycling was carried out on the EOT samples. CV measurements were taken in-situ at 0,10 , $100,1000,3000,10,000$ and 16,000 cycles to monitor the degradation in the ECSA over the course of the AST. Polarization curves were collected at BOT and EOT.

Microstructural characterization.-X-ray computed tomography (X-ray CT) was carried out using a Zeiss Xradia 520 Versa (Carl Zeiss X-ray Microscopy Inc., Pleasanton, CA). A biopsy punch was used to cut a disc with a diameter of $1 \mathrm{~mm}$ from a channel region of each sample, then mounted on to a pinhead, with the cathode side of the MEA in the top of the field of view (FOV) for each scan. Tomographic scans were retrieved by the collection of 3701 radiograph projections through a $360^{\circ}$ rotation of the sample. For each sample, an X-ray source voltage of $80 \mathrm{kV}$ was used with a $12 \mathrm{~s}$ exposure time and a $0.7 \mathrm{~mm} \times 0.7 \mathrm{~mm}$ square FOV. Reconstruction was carried out using a commercial image reconstruction software package (Zeiss XMReconstructor, Carl Zeiss X-ray Microscopy Inc., Pleasanton, CA), which employs a cone-beam filtered back projection (FBP) algorithm. Resulting tomogram pixel sizes were $343 \mathrm{~nm}, 389$ $\mathrm{nm}, 343 \mathrm{~nm}$ and $366 \mathrm{~nm}$ for HP_BOT, NHP_BOT, HP_EOT and NHP_EOT data sets, respectively. The X-ray imaging conditions are summarized in Table II.

Scanning electron microscopy was carried out to characterize the CL surface of a fresh GDE. A disc of the electrode with a diameter of $5 \mathrm{~mm}$ was punched out and adhered to carbon tape on an SEM stub. Imaging was done using the Zeiss SEM EVO MA 10 (Carl Zeiss, USA), with an accelerating voltage of $10 \mathrm{kV}$, a working distance of
$8.0 \mathrm{~mm}$ and a pixel size of between $2.2 \mu \mathrm{m}$ and $0.9 \mu \mathrm{m}$, depending on the magnification of the image.

X-ray CT image post-processing.-Post-processing was carried out using Avizo software (Avizo, Thermo Fisher Scientific, Waltham Massachusetts, USA), by cropping each dataset to a $400 \mu \mathrm{m} \times 400$ $\mu \mathrm{m} \times 345 \mu \mathrm{m}$ interfacial subvolume. Each dataset was then manually segmented into five phases using the 'magic wand' tool according to the material grayscale values: pore, GDLs, cathode CL, Nafion and anode CL. The GDL phases consist of all fibers, PTFE additive and MPL, since the similarity of grayscale values of the MPL and fibers meant that segmentation of these two phases was not possible. Because the CLs are the main focus of this study, the GDLs were not separated into the constituent anode and cathode GDL phases. The 3D structures of the materials were visualized using volume renderings of the segmented phases, to give an appreciation of the layers of the MEA in each sample. These are accompanied by solidpore percentage compositions quantified by the average volume of a slice-by-slice analysis of each material, in each sample, to ensure sufficient statistical representation. The analysis was done using a materials statistics analysis of the volume-per-slice, then averaging using the following,

$$
V_{f}=\frac{\sum_{i} V_{j}}{V_{t}} \times 100 \%
$$

where $V_{f}$ is the volume percent in $\%, i$ is the number of slices, $V_{j}$ is the volume of phase $j$ in $\mu \mathrm{m}^{3}$, which represents the sum of all voxels belonging to phase $j$ in any given orthoslice, and $V_{t}$ is the total volume of the interfacial subvolume in $\mu \mathrm{m}^{3}$.

To calculate the normalized interfacial contact areas, with no units, between the cathode CL-Nafion and anode CL-Nafion, the method described in previous work by the authors was used. ${ }^{20}$ The method involves creating a surface, followed by a Surface Area Analysis, finally normalizing to the surface area of one slice.

Since the AST was cathode CL-specific, further post-processing analyses were carried out on the cathode CL, only. The phases fibers, Nafion and anode CL, were removed from the volume rendering visualization and a further phase, namely "cracks" was segmented in each sample. The newly segmented image was duplicated and all phases except cracks (fibers, Nafion, anode CL and cathode CL) were removed, such that the crack-analysis was only carried out on material associated with the "cracks" phase. Analysis of the cracks was done using a distance-ordered homeotropic thinning (DOHT) method, which is used to calculate the local thickness at points throughout a crack skeleton. DOHT is a well-established algorithm for this sort of analysis, which has been discussed thoroughly elsewhere. ${ }^{39}$

The average indentation diameter of the NHP_BOT sample was found by measuring the diameter of all indentations in the raw X-ray CT data set, then averaging all values. Similarly, the average indentation diameter of the fresh GDE was calculated by averaging diameter measurements using the SEM image with $129 \times$ magnification.

\section{Results and Discussion}

Electrochemical characterization.-The key findings of the electrochemical characterization are summarized in Table III. Polarization curves were measured for the conditioned HP_BOT and NHP_BOT MEAs (Figure 2a). Comparison of the BOT curves shows a negligible

Table II. X-ray imaging conditions for the four PEFC samples, characterized using the Zeiss Xradia 520 Versa X-ray CT instrument.

\begin{tabular}{|c|c|c|c|c|}
\hline Sample & HP_BOT & NHP_BOT & HP_EOT & NHP_EOT \\
\hline Isotropic resolution/nm & 343 & 389 & 343 & 366 \\
\hline $\mathrm{X}$-ray volume $/ \mu \mathrm{m} \times \mu \mathrm{m} \times \mu \mathrm{m}$ & \multicolumn{4}{|c|}{$700 \times 700 \times 700$} \\
\hline Interfacial sub-volume $/ \mu \mathrm{m} \times \mu \mathrm{m} \times \mu \mathrm{m}$ & \multicolumn{4}{|c|}{$400 \times 400 \times 389$} \\
\hline Cathode CL sub-volume $/ \mu \mathrm{m} \times \mu \mathrm{m} \times \mu \mathrm{m}$ & \multicolumn{4}{|c|}{$400 \times 400 \times 66$} \\
\hline Projections/no-units & \multicolumn{4}{|c|}{3701} \\
\hline Exposure time/seconds & \multicolumn{4}{|c|}{12} \\
\hline
\end{tabular}


Table III. Summary of the electrochemical data for the four samples, HP_BOT, NHP_BOT, HP_EOT and NHP_EOT obtained from polarization curves and electrochemical surface area analyses.

\begin{tabular}{|c|c|c|c|c|}
\hline Sample & HP_BOT & NHP_BOT & HP_EOT & NHP_EOT \\
\hline $\mathrm{OCV} / \mathrm{V}$ & 0.96 & 0.95 & 0.94 & 0.92 \\
\hline Onset of ohmic loss region/ $\mathrm{mA} \mathrm{cm}-2$ & 30 & 30 & 19 & 19 \\
\hline Onset of mass transport region $/ \mathrm{mA} \mathrm{cm} \mathrm{cm}^{-2}$ & 680 & 680 & 662 & 662 \\
\hline Peak power density $/ \mathrm{mW} \mathrm{cm}^{-2}$ & 361 & 376 & 311 & 318 \\
\hline $\mathrm{ECSA} / \mathrm{m}^{2} \mathrm{~g}_{\mathrm{Pt}^{-1}}$ & 53.3 & 49.8 & 12.9 & 13.7 \\
\hline
\end{tabular}

difference in performance for both the hot-pressed and self-assembled MEAs, with a limiting current density of $\sim 775 \mathrm{~mA} \mathrm{~cm}^{-2}$ at $0.2 \mathrm{~V}$. This is contrary to literature research, which states that an MEA with no hot pressing shows worse performance. ${ }^{21}$ In both cases, onset of the ohmic loss region is at $\sim 30 \mathrm{~mA} \mathrm{~cm}^{-2}$ and onset of the mass transport region is at $\sim 680 \mathrm{~mA} \mathrm{~cm}^{-2}$. Furthermore, the power density curves show a peak power density of $361 \mathrm{~mW} \mathrm{~cm}^{-2}$ for the HP_BOT and a slightly higher value of $376 \mathrm{~mW} \mathrm{~cm}^{-2}$ for the NHP_BOT sample. For both samples, the peak power density occurs at a current density of $624 \mathrm{~mA} \mathrm{~cm}^{-2}$.

After the AST (16,000 cycles), a drop in cell performance was observed in the polarization curves of both the hot-pressed and selfassembled samples (Figure 2a). The extent to which the samples have degraded is almost identical, the majority of which appears to have occurred in the kinetic region. This implies that the MEA degrades at the same rate, regardless of whether it has been hot-pressed or not.
A small drop in OCV was observed for both samples, from $0.96 \mathrm{~V}$ to $0.94 \mathrm{~V}$ for the hot-pressed MEA and from $0.95 \mathrm{~V}$ to $0.92 \mathrm{~V}$ for the self-assembled MEA. Onset of the ohmic loss region occurred at $19 \mathrm{~mA} \mathrm{~cm}{ }^{-2}$ for the EOT samples, which is around half of the BOT onset value. A steeper gradient in the activation loss region is consistent with the cathode electrocatalyst-specific AST used.

Comparison of the BOT cyclic voltammograms shows the hydrogen/Pt-O adsorption and desorption peaks during the forward and reverse voltage sweeps (Figure $2 b$ ). ${ }^{37}$ For the self-assembled MEA, the hydrogen adsorption/desorption peaks are slightly smaller than the hot-pressed MEA, which is characterized by ECSA values of $49.8 \mathrm{~m}^{2} \mathrm{~g}_{\mathrm{Pt}}{ }^{-1}$ and $53.3 \mathrm{~m}^{2} \mathrm{~g}_{\mathrm{Pt}}{ }^{-1}$ for NHP_BOT and HP_BOT, respectively. Despite the slightly lower ECSA of the NHP_BOT sample, the $\mathrm{CV}$ profiles of the two BOT samples further supports the findings of polarization curves that performance is almost identical regardless of the MEA preparation technique.

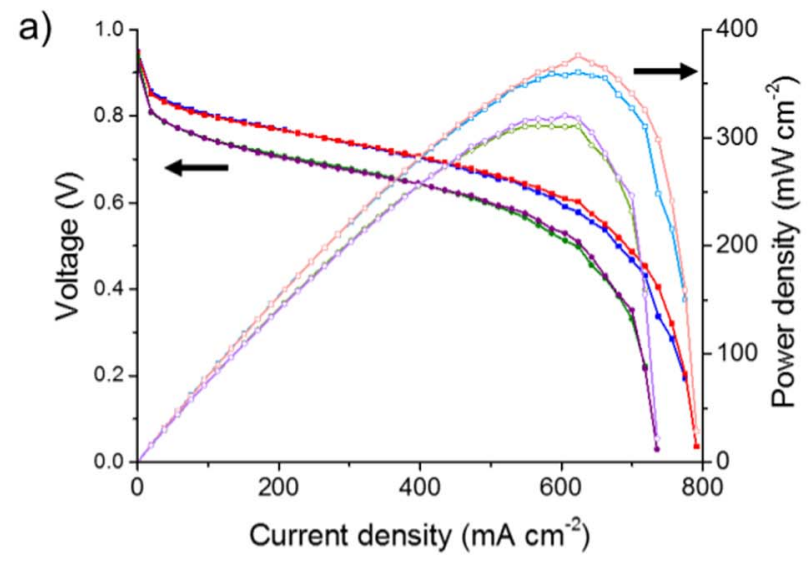

$\longrightarrow$ HP130_BOT $\longrightarrow$ NHP_BOT $\longrightarrow$ HP_EOL $\longrightarrow$ NHP_EOT

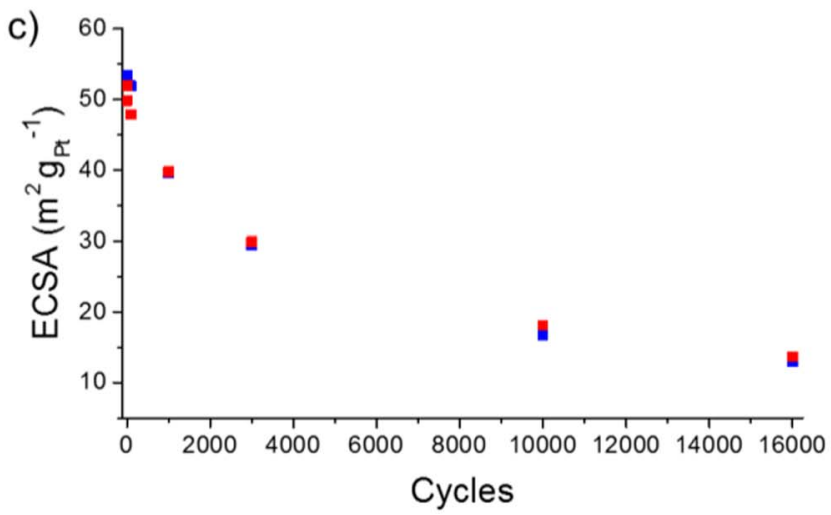

- Hot-pressed . Non-hot-pressed b)

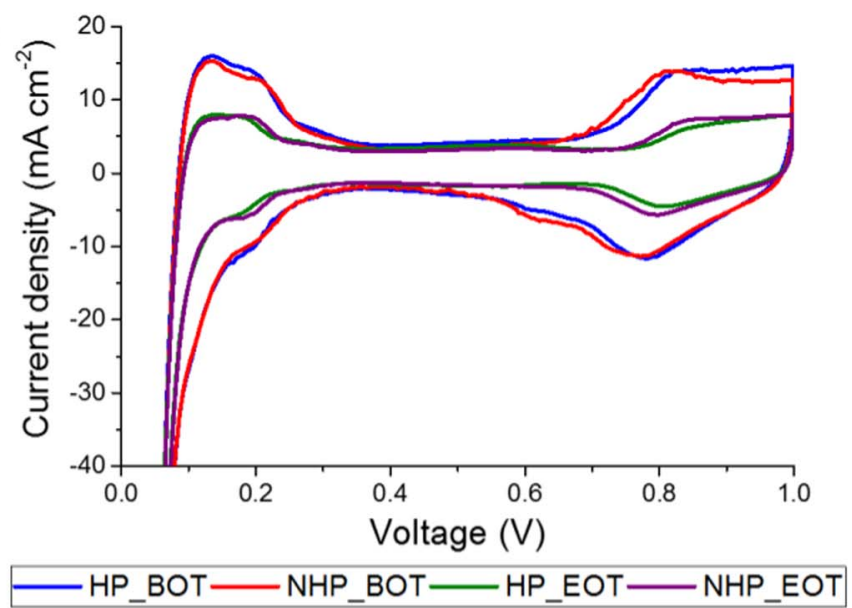

d)

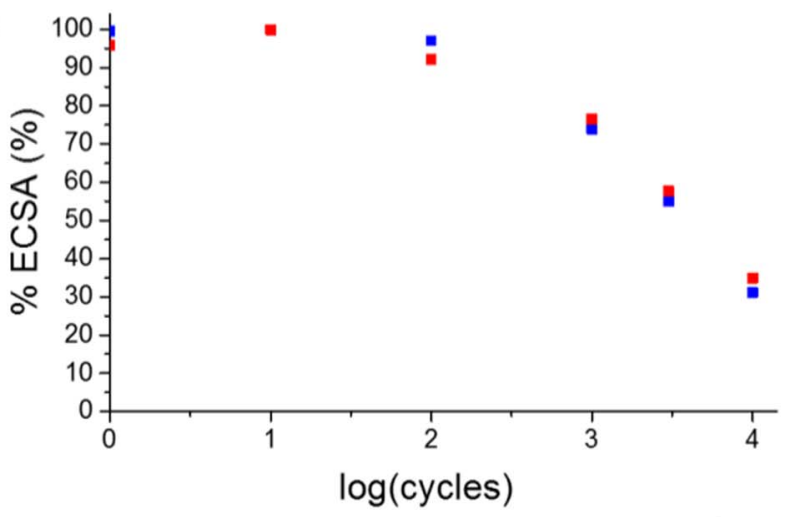

- Hot-pressed . Non-hot-pressed

Figure 2. a) BOT and EOT polarization curves (dark, closed symbols) and power density curves (light, open symbols) for the HP_BOT (blue), NHP_BOT (red), HP_EOT (green) and NHP_EOT (purple), b) cyclic voltammograms for HP_BOT (blue), NHP_BOT (red), HP_EOT (green) and NHP_EOT (purple), c) decrease in the ECSA over the course of cycling for hot-pressed (blue) and self-assembled (red) MEAs and d) the associated percentage of initial ECSA for hot-pressed (blue) and self-assembled (red). 


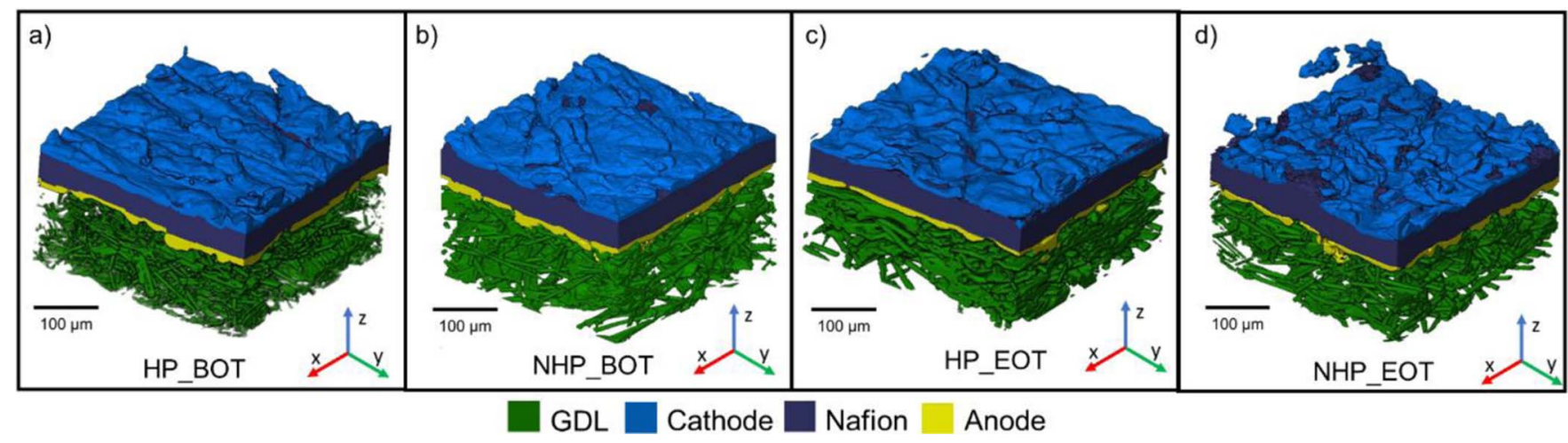

Figure 3. Surface rendering of a) HP_BOT, b) NHP_BOT, c) HP_EOT and d) NHP_EOT samples. Each layer is labeled according to the legend, with the cathode $\mathrm{CL}$ at the top and anode $\mathrm{CL}$ on the bottom for each sample. The $\mathrm{z}$ direction is indicated in each instance, to highlight the viewing perspective shown in Figures $4 \mathrm{a}-4 \mathrm{~d}$.

EOT voltammograms (Figure 2b) show that the area under all of the adsorption and desorption peaks has reduced for both samples, which is attributed to a loss of catalytically active sites as a result of the CL-specific AST. At EOT the self-assembled MEA has a slightly higher ECSA of $13.7 \mathrm{~m}^{2} \mathrm{~g}_{\mathrm{Pt}^{-1}}{ }^{-1}$, compared with $12.9 \mathrm{~m}^{2} \mathrm{~g}_{\mathrm{Pt}^{-1}}$ for the hot-pressed sample.

In-situ CV was carried out, as described in Materials section, and a plot of ECSA against cycle number shows a reduction in the ECSA for both samples ${ }^{40}$ (Figure 2c). An increase in the ECSA of $4 \%$ is observed in the first 10 cycles of the self-assembled sample. This is attributed to cleaning of the Pt-surface, which has been described for other measurements of in-situ ECSA. ${ }^{21}$ The percentage of the initial ECSA was also calculated for both samples and it was found that the EOT ECSA was $27.5 \%$ of the initial ECSA for NHP_EOT and $24.2 \%$ of the initial values for HP_BOT (Figure 2d). While ECSA values depend on both the chosen AST and the materials used, the values calculated here are comparable with those that have been reported previously. ${ }^{21,41,42}$ Once again, the durability of the MEA is shown to be independent of MEA preparation technique.

$X$-ray computed tomography.-Although the HP and NHP MEAs show no difference in performance, it was suspected that the MEA microstructure would respond differently to the AST due to the different preparation methods used. Therefore, X-ray CT was use to compare the hot-pressed and self-assembled structure both before and after the AST had been carried out. A volume rendering of each sample was created (Figures 3a-3d), to allow the distinct phases to be visualized and to enable further quantitative analysis. Anode GDLs have been shown to emphasize the composition of the layers in the MEA and it should be noted that there is a similar GDL above the cathode CL, which has not been shown in these images. This allows for the top surface of the cathode CL to be visualized. For the EOT samples, such a perspective highlights the deterioration of the cathode CL in both cases post-AST (Figure 3c and 3d for HP_EOT and NHP_EOT, respectively). For example, the delamination of the CL is particularly obvious in the NHP_EOT sample (Figure 3d) and the protrusion of the blue cathode CL further in the positive z-direction indicates that the $\mathrm{CL}$ is starting to impregnate into the GDL.

A solid-phase volume percentage analysis was carried out on each sample to compare the proportion of each material in the subvolume

\begin{abstract}
Table IV. Solid-phase volume percentage compositions of each material layer within four MEA samples obtained from laboratorybased X-ray computed tomography. In each case, $i$ refers to the solid-phase variable being described: either CCL, Nafion or ACL, for cathode CL, Nafion and anode CL, respectively.
\end{abstract}

\begin{tabular}{lcccc} 
Sample & HP_BOT & NHP_BOT & HP_EOT & NHP_EOT \\
\hline Figure 3 & $\mathrm{a}$ & $\mathrm{b}$ & $\mathrm{c}$ & $\mathrm{d}$ \\
& $V_{H P, B O T, i}$ & $V_{N H P, B O T, i}$ & $V_{H P, E O T, i}$ & $V_{N H P, E O T, i}$ \\
Cathode CL/\% & $2.9 \pm 0.4$ & $2.9 \pm 0.3$ & $2.4 \pm 0.3$ & $2.2 \pm 0.4$ \\
Nafion/\% & $13.2 \pm 0.5$ & $15.2 \pm 0.3$ & $13.1 \pm 0.3$ & $14.9 \pm 0.5$ \\
Anode CL/\% & $3.1 \pm 0.4$ & $3.0 \pm 0.4$ & $2.7 \pm 0.2$ & $2.7 \pm 0.3$
\end{tabular}

(Table IV). The Nafion content in the hot-pressed samples was found to be lower than for the self-assembled samples, with values of $13.2 \%$ for $V_{H P, B O T, N a f i o n}$ and $13.1 \%$ for $V_{H P, E O T, N a f i o n}$, compared with $14.9 \%$ for $V_{N H P, B O T, N a f i o n}$ and $15.2 \%$ for $V_{N H P, E O T, N a f i o n}$. This disparity could arise due to the additional compression from the hot-press platens that is experienced by the hot-pressed MEAs, which would lead to a more compressed layer occupying a smaller amount of the volume.

Regarding the CL solid-phase volume percentages, the BOT values were higher than EOT values for both samples, which suggests that the AST has resulted in an irreversible effect on the CL over the course of cycling. Volume percentage loss from the cathode CL of each sample was greater than volume loss from the anode CL, which is in agreement with the cathode-specific AST that was carried out. A $0.5 \%$ loss was observed for the hot-pressed cathode CL, compared with a $0.7 \%$ loss in the volume of the self-assembled cathode CL, which highlights the similar extent of degradation of the hot-pressed and self-assembled samples.

Analysis of the interfacial normalized contact area was carried out on all samples to analyze the interfaces between the electrolyte and each electrode (Table V). For both the hot-pressed and self-assembled samples, the interfacial contact area was found to decrease. The loss in contact between the Nafion and CL supports the findings of the volume percentage compositions that there has been some irreversible effect on the CL as a result of the AST. Interfacial contact of the NHP_BOT sample was found to be lower for both the anode and cathode than the HP_BOT, which suggests that the hot-pressed sample is in closer

Table V. Interfacial normalized contact area (CA) obtained from laboratory-based X-ray computed tomography. In each case, $j$ refers to the interface being described: CCL Nafion or ACL-Nafion.

\begin{tabular}{|c|c|c|c|c|}
\hline Sample & HP_BOT & NHP_BOT & HP_EOT & NHP_EOT \\
\hline Figure 3 & $\mathrm{a}$ & $\mathrm{b}$ & $\mathrm{c}$ & $\mathrm{d}$ \\
\hline & $C A_{H P, B O T, j}$ & $C A_{N H P, B O T, j}$ & $C A_{H P, E O T, j}$ & $C A_{N H P, E O T, j}$ \\
\hline Cathode CL-Nafion/no-units & $1.16 \pm 0.01$ & $0.91 \pm 0.02$ & $0.82 \pm 0.03$ & $0.78 \pm 0.05$ \\
\hline Anode CL-Nafion/no-units & $1.05 \pm 0.02$ & $0.98 \pm 0.02$ & $0.89 \pm 0.04$ & $0.77 \pm 0.05$ \\
\hline
\end{tabular}




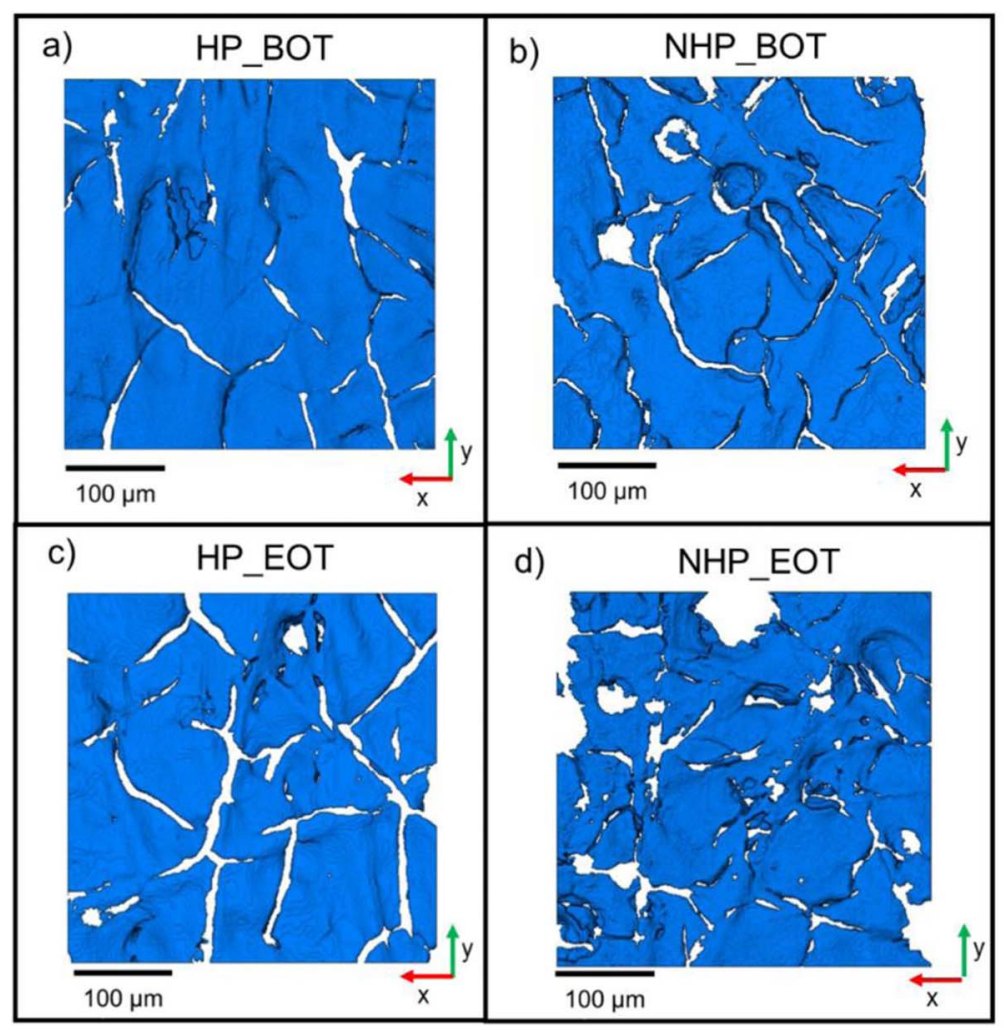

e)

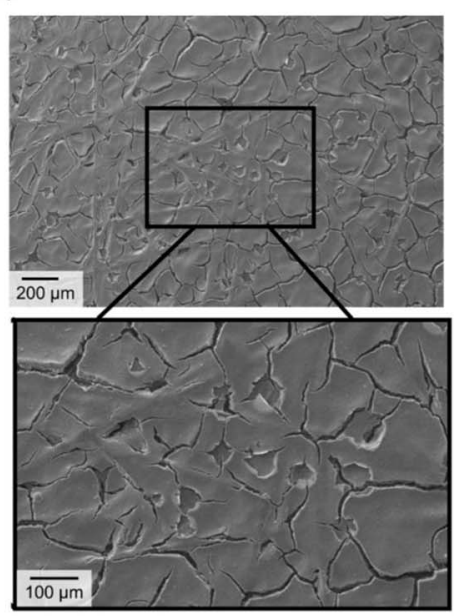

Figure 4. Volume-rendered image of the isolated cathode CL for a) HP_BOT, b) NHP_BOT, c) HP_EOT and d) NHP_EOT. In each case, the perspective is looking along the z-axis. e) SEM image, with $129 \times$ magnification (top) and $325 \times$ magnification (bottom) of the fresh GDE, looking onto the surface of the CL. The crack network is seen, as well as the dimples that arise from popped bubbles that occur during the drying process.

contact with the Nafion as a result of the hot-pressing process. Furthermore, at EOT, the hot-pressed contact area is slightly higher than the self-assembled contact area, which also indicates a difference in microstructure between the hot-pressed and self-assembled MEAs.

Since X-ray CT is a non-destructive technique, visualization of interfaces in the MEA that are normally hidden is possible. As well as information about solid-phase volume fraction, segmentation of the sample into its constituent phases allows for isolation and analysis of the individual materials. For each sample, the cathode CL of the tomogram was isolated and viewed from the CL underside, i.e. from the perspective of the Nafion looking at the Nafion-CL interface (Figures $4 \mathrm{a}-4 \mathrm{~d}$ ).

Using the isolated images, it was possible to segment the 3D crack network for each cathode CL, which allowed for detailed analysis of the crack properties of each sample. Using the DOHT method that was described in Microstructural characterization section, a 3D map of the cracks in the CL was created (Figures 5a-5d). The skeleton of the crack network is shown in Figure 5 for each sample and the color of the skeleton corresponds to the normalized thickness, according to the scale shown.

The thickness of the cracks was calculated at discrete points throughout the crack network, which allowed for calculation of the average crack width for each sample (Table VI). For the hot-pressed sample, a BOT crack width of $6 \pm 1 \mu \mathrm{m}$ was found, which is in good agreement with other examples of crack width investigations. ${ }^{20,42,43}$ In addition, crack widths of an SEM image of a fresh ELE0162 GDE were measured (Figure 4e), giving an average crack width of $7 \pm 2$ $\mu \mathrm{m}$, which again supports the findings of the X-ray CT data.

At EOT, the crack-width in the hot-pressed sample was found to increase to an average value of $9 \pm 1 \mu \mathrm{m}$, which indicates that the cracks have widened over the course of the AST. The mechanism of crack-widening resulting from an AST has been previously reported $^{42}$ and the values for crack widths at BOT and EOT are in good agreement with the experimental results found here. Regard- ing the self-assembled sample, only a marginal increase in the crack width was observed, from $6 \pm 1 \mu \mathrm{m}$ to $7 \pm 1 \mu \mathrm{m}$ for NHP_BOT and NHP_EOT, respectively. A possible reason for this could be that the self-assembled CL degrades via an alternative degradation process, which will be discussed further in the following section.

Rather than expansion of the crack network, which appears to be characteristic of the hot-pressed degradation, it is proposed that the self-assembled CL degrades by expansion of a number of 'indentations' that are observed in the surface of the self-assembled CL. From analysis of the cathode CL volume renderings (Figures $4 \mathrm{~b}$ and $4 \mathrm{~d}$ for NHP_BOT and NHP_EOT, respectively), it can be seen that there are a number of indentations spaced irregularly over the surface of the BOT and EOT catalyst layers. On the other hand, no indentations are observed on the hot-pressed samples. Furthermore, an SEM image of the CL surface of a fresh ELE0162 GDE shows clearly that these indentations are present over the whole surface of the starting material (Figure 4e).

Whilst cracks in the CL have previously been discussed in the literature,,$^{20,42}$ to the authors' knowledge, the presence of indentations in the CL has not yet been reported. The indentations, which are, as discussed, also present in the CL starting material, are attributed to solvent bubbles in the CL ink that burst during drying once the CL has been coated onto the MPL. Measurement of the indentation diameter for the $129 \times$ image (Figure 4, top) found an average diameter of $48 \pm 13 \mu \mathrm{m}$, which is in agreement with the average value of $47 \mu \mathrm{m}$ found for the NHP_BOT sample measured from the X-ray CT data.

Thus, it is suggested that the microstructure of the CL in the hot-pressed and self-assembled MEAs is affected by the preparation technique used, in the following way (Figure 1).

- In Step 1, the fresh starting GDE electrodes have indentations and cracks present.

- During the hot-pressing step (Step 2), the indentations are "smoothed out" by the applied temperature and pressure of the platens. 


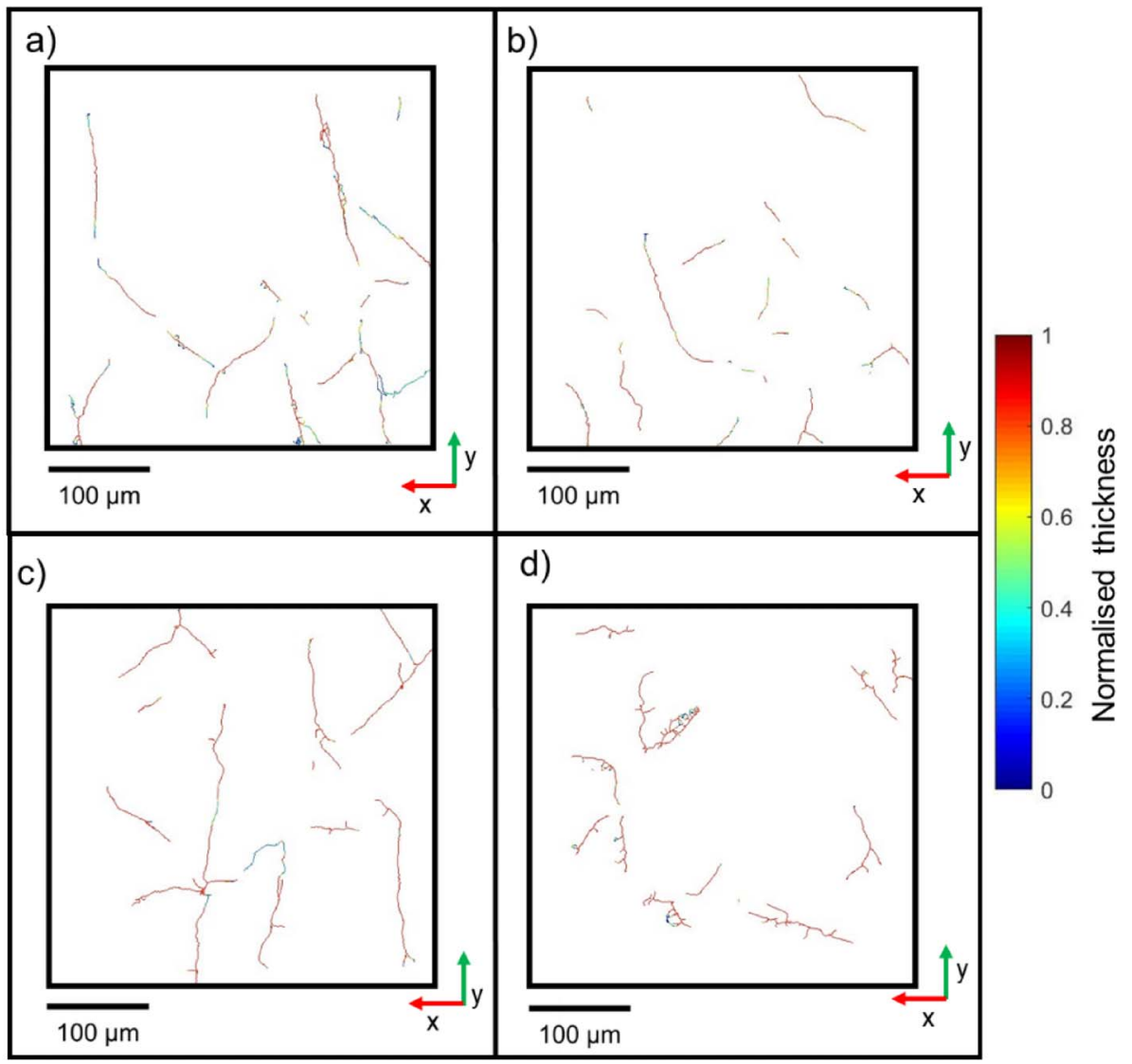

Figure 5. 3D skeleton crack networks for the four samples, a) HP_BOT, b) NHP_BOT, c) HP_EOT and d) NHP_EOT, with the normalized thickness gradient shown by the color bar.

$\begin{aligned} & \text { Table VI. Average crack diameter for each sample, calculated by } \\
& \text { averaging the thickness calculated using the DOHT method. }\end{aligned}$
\begin{tabular}{lccccc} 
Sample & HP_BOT & NHP_BOT & HP_EOT & NHP_EOT \\
\hline $\begin{array}{l}\text { Figure 5 } \\
\text { Average crack width/ } \mu \mathrm{m}\end{array}$ & $6 \pm 1$ & $6 \pm 1$ & $9 \pm 1$ & $7 \pm 1$
\end{tabular}

The non-hot-press route proceeds directly to the conditioning of the MEAs in Step 3.

- In Step 4, after conditioning and prior to AST, the samples are removed from the test cell and X-ray CT is carried out, giving HP_BOT and NHP_BOT. It is observed that the temperature and torque of the fuel cell housing after conditioning are sufficient to laminate the GDEs to the Nafion in the NHP_BOT sample. However, the conditions are not sufficient to "smooth out" the indentations.

- Steps 1-3 are repeated for two fresh samples and now the AST is carried out directly after conditioning in Step 4, to produce two further, degraded samples, HP_EOT and NHP_EOT. The NHP_EOT sample still has indentations in the CL microstructure, which have widened, whereas the HP_EOT sample proceeds via crack widening degradation.

\section{Conclusions}

This study has demonstrated the use of correlative electrochemical/X-ray CT characterization to inspect the difference between hot-pressed and self-assembled MEAs. It was found that there was no significant difference between the hot-pressed and self-assembled MEA performance and the AST resulted in degradation at the same rate for both systems, as shown by the similarity in the EOT performance and ECSA at EOT.

$\mathrm{X}$-ray CT was used to further investigate the microstructural features of the hot-pressed and self-assembled MEAs. Results of solid- phase volume fraction analyses and interfacial contact area calculations were found to decrease from BOT to EOT, for both preparation methods. This suggested that degradation rate of the hot-pressed and self-assembled samples was similar, which was also indicated by electrochemical testing.

Inspection of the CL gave novel insight into differences in the hotpressed and self-assembled microstructure. Whereas the hot-pressed sample had a structure consisting of a crack network, only, the selfassembled sample was found to have additional indentations as well as a network of cracks. The indentations were found to be present in the starting material, as characterized by SEM of the surface of a fresh GDE. After the AST had been carried out, it was found that the cracks in the hot-pressed sample had widened. However, it is thought that the self-assembled sample degrades by an expansion of the indentations, which provides scope for further investigation of this phenomenon in future studies.

Finally, the similarity in performance and durability of the two samples presents an argument for the by-passing of the hot-pressing step in favor of an in-situ assembly of MEAs. This would constitute both a time and cost saving, when considering the overall, PEFC life-cycle.

\section{Acknowledgments}

Jennifer Hack acknowledges a studentship from the EPSRC CDT for the Advanced Characterization of Materials (EP/LO15277/1). The authors also acknowledge funding from the EPSRC (EP/P009050/1, EP/M014371/1, EP/M009394/1, EP/M023508/1, EP/L015749/1, EP/N022971/1) for supporting this research.

\section{ORCID}

\section{J. L. Brett (1D https://orcid.org/0000-0002-8545-3126}




\section{References}

1. A. Kirubakaran, S. Jain, and R. K. Nema, "A review on fuel cell technologies and power electronic interface," Renew. Sustain. Energy Rev., 13, 2430 (2009).

2. S. Evangelisti, C. Tagliaferri, D. J. L. Brett, and P. Lettieri, "Life cycle assessment of a polymer electrolyte membrane fuel cell system for passenger vehicles," J. Clean. Prod., 142, 4339 (2017)

3. S. J. Peighambardoust, S. Rowshanzamir, and M. Amjadi, "Review of the proton exchange membranes for fuel cell applications," Int. J. Hydrogen Energy., 35, 9349 (2010).

4. J. M. Song, S. Y. Cha, and W. M. Lee, "Optimal composition of polymer electrolyte fuel cell electrodes determined by the AC impedance method," J. Power Sources. 94, 78 (2001).

5. V. A. Paganin, E. A. Ticianelli, and E. R. Gonzalez, "Development and electrochemical studies of gas diffusion electrodes for polymer electrolyte fuel cells," J. Appl. Electrochem., 26, 297 (1996).

6. X. Zhang, Y. Gao, H. Ostadi, K. Jiang, and R. Chen, "Modelling water intrusion and oxygen diffusion in a reconstructed microporous layer of PEM fuel cells," Int. J. Hydrogen Energy., 39, 17222 (2014).

7. W. Mróz, B. Budner, W. Tokarz, P. Piela, and M. L. Korwin-Pawlowski, "Ultralow-loading pulsed-laser-deposited platinum catalyst films for polymer electrolyte membrane fuel cells," J. Power Sources., 273, 885 (2015).

8. M. Breitwieser, M. Klingele, B. Britton, S. Holdcroft, R. Zengerle, and S. Thiele, "Improved Pt-utilization efficiency of low Pt-loading PEM fuel cell electrodes using direct membrane deposition," Electrochem. Commun., 60, 168 (2015).

9. V. Mehta and J. S. Cooper, "Review and analysis of PEM fuel cell design and manufacturing," J. Power Sources., 114, 32 (2003)

10. M. S. Wilson and S. Gottesfeld, "High Performance Catalyzed Membranes of Ultralow Pt Loadings for Polymer Electrolyte Fuel Cells,” J. Electrochem. Soc., 139, L28 (1992).

11. M. Prasanna, E. A. Cho, T. H. Lim, and I. H. Oh, "Effects of MEA fabrication method on durability of polymer electrolyte membrane fuel cells," Electrochim. Acta., 53, 5434 (2008).

12. S. Prass, S. Hasanpour, P. K. Sow, A. B. Phillion, and W. Mérida, "Microscale X-ray tomographic investigation of the interfacial morphology between the catalyst and micro porous layers in proton exchange membrane fuel cells," J. Power Sources., 319, 82 (2016)

13. S. Kundu, M. W. Fowler, L. C. Simon, and S. Grot, "Morphological features (defects) in fuel cell membrane electrode assemblies," J. Power Sources., 157, 650 (2006).

14. E. A. Ticianelli, C. R. Derouin, A. Redondo, and S. Srinivasan, "Methods to Advance Technology of Proton Exchange Membrane Fuel Cells," J. Electrochem. Soc., 135, 2209 (1983).

15. H. K. Lee, J. H. Park, D. Y. Kim, and T. H. Lee, "A study on the characteristics of the diffusion layer thickness and porosity of the PEMFC," J. Power Sources., 131, 200 (2004).

16. H. Tang, S. Wang, S. P. Jiang, and M. Pan, "A comparative study of CCM and hot-pressed MEAs for PEM fuel cells,” J. Power Sources., 170, 140 (2007).

17. E. Negro, R. Latsuzbaia, M. Dieci, I. Boshuizen, and G. J. M. Koper, "Pt electrodeposited over carbon nano-networks grown on carbon paper as durable catalyst for PEM fuel cells," Appl. Catal. B Environ., 166-167, 155 (2015).

18. B. Millington, V. Whipple, and B. G. Pollet, "A novel method for preparing proton exchange membrane fuel cell electrodes by the ultrasonic-spray technique," J. Power Sources., 196, 8500 (2011).

19. B. Millington, S. Du, and B. G. Pollet, "The effect of materials on proton exchange membrane fuel cell electrode performance," J. Power Sources., 196, 9013 (2011)

20. Q. Meyer, N. Mansor, F. Iacoviello, P. L. Cullen, R. Jervis, D. Finegan, C. Tan, J. Bailey, P. R. Shearing, and D. J. L. Brett, "Investigation of Hot Pressed Polyme Electrolyte Fuel Cell Assemblies via X-ray Computed Tomography," Electrochim. Acta., 242, 125 (2017).

21. S. M. Andersen, R. Dhiman, M. J. Larsen, and E. Skou, "Importance of electrode hot-pressing conditions for the catalyst performance of proton exchange membrane fuel cells," Appl. Catal. B Environ., 172-173, 82 (2015).

22. A. Bayrakceken, S. Erkan, L. Turker, and I. Eroglu, "Effects of membrane electrode assembly components on proton exchange membrane fuel cell performance," Int. J. Hydrogen Energy., 33, 165 (2008).
23. R. Zeis, A. Mathur, G. Fritz, J. Lee, and J. Erlebacher, "Platinum-plated nanoporous gold: An efficient, low Pt loading electrocatalyst for PEM fuel cells," J. Power Sources., 165, 65 (2007).

24. X. Yin, L. Lin, H. T. Chung, S. Komini Babu, U. Martinez, G. M. Purdy, and P. Zelenay, "Effects of MEA Fabrication and Ionomer Composition on Fuel Cell Performance of PGM-Free ORR Catalyst," ECS Trans., 77, 1273 (2017).

25. A. Therdthianwong, P. Manomayidthikarn, and S. Therdthianwong, "Investigation of membrane electrode assembly (MEA) hot-pressing parameters for proton exchange membrane fuel cell," Energy, 32, 2401 (2007).

26. Y. Tang, J. Zhang, C. Song, H. Liu, J. Zhang, H. Wang, S. Mackinnon, T. Peckham, J. Li, S. McDermid, and P. Kozak, "Temperature Dependent Performance and In Situ AC Impedance of High-Temperature PEM Fuel Cells Using the Nafion-112 Membrane," J. Electrochem. Soc., 153, A2036 (2006).

27. S. Martemianov, V. A. Raileanu Ilie, and C. Coutanceau, "Improvement of the proton exchange membrane fuel cell performances by optimization of the hot pressing process for membrane electrode assembly," J. Solid State Electrochem., 18, 1261 (2014).

28. S. Zhang, X. Yuan, H. Wang, W. Mérida, H. Zhu, J. Shen, S. Wu, and J. Zhang, "A review of accelerated stress tests of MEA durability in PEM fuel cells," Int. J. Hydrogen Energy, 34, 388 (2009).

29. C. Lim, L. Ghassemzadeh, F. Van Hove, M. Lauritzen, J. Kolodziej, G. G. Wang, S. Holdcroft, and E. Kjeang, "Membrane degradation during combined chemical and mechanical accelerated stress testing of polymer electrolyte fuel cells," J. Power Sources., 257, 102 (2014).

30. R. Petrone, D. Hissel, M. C. Péra, D. Chamagne, and R. Gouriveau, "Accelerated stress test procedures for PEM fuel cells under actual load constraints: State-of-art and proposals," Int. J. Hydrogen Energy., 40, 12489 (2015).

31. I. V Zenyuk, D. Y. Parkinson, G. Hwang, and A. Z. Weber, "Probing water distribution in compressed fuel-cell gas-diffusion layers using X-ray computed tomography," Electrochem. Commun., 53, 24 (2015).

32. R. T. White, F. P. Orfino, M. El Hannach, O. Luo, M. Dutta, A. P. Young, and E. Kjeang, "3D Printed Flow Field and Fixture for Visualization of Water Distribution in Fuel Cells by X-ray Computed Tomography," J. Electrochem. Soc., 163, F1337 (2016).

33. A. Pfrang, S. Didas, and G. Tsotridis, "X-ray computed tomography of gas diffusion layers of PEM fuel cells: Segmentation of the microporous layer," J. Power Sources., 235, 81 (2013).

34. W. K. Epting, J. Gelb, and S. Litster, "Resolving the Three-Dimensional Microstructure of Polymer Electrolyte Fuel Cell Electrodes using Nanometer-Scale X-ray Computed Tomography," Adv. Funct. Mater, 22, 555 (2012).

35. K. M. Ø. Jensen, X. Yang, J. V. Laveda, W. G. Zeier, K. A. See, M. Di Michiel, B. C. Melot, S. A. Corr, and S. J. L. Billinge, "X-Ray Diffraction Computed Tomography for Structural Analysis of Electrode Materials in Batteries," J. Electrochem. Soc., 162, A1310 (2015)

36. Fuel Cells Technologies Office Multi-Year Research, Development, and Demonstration Plan - Section 3.4 Fuel Cells, (n.d.). https://www.energy.gov/sites/ prod/files/2016/06/f32/fcto myrdd fuel_cells.pdf (accessed December 6, 2017).

37. J. Wu, X.-Z. Yuan, and H. Wang, Cyclic Voltammetry, in: PEM Fuel Cell Diagnostic Tools, CRC Press, 2011: pp. 71.

38. U.S. DRIVE Fuel Cell Tech Team, Fuel Cell Tech Team AST and Polarization Curve Protocols for PEMFCs, U.S. DRIVE Fuel Cell Tech Team. (2013). https://energy.gov/sites/prod/files/2015/08/f25/fcto_dwg_usdrive_fctt_accelerated_ stress tests jan2013.pdf (accessed December 6, 2017).

39. O. O. Taiwo, D. P. Finegan, D. S. Eastwood, J. L. Fife, L. D. Brown, J. A. Darr, P. D. Lee, D. J. L. Brett, and P. R. Shearing, "Comparison of three-dimensional analysis and stereological techniques for quantifying lithium-ion battery electrode microstructures," J. Microsc., 263, 280 (2016).

40. D. Bezmalinovic, B. Simic, and F. Barbir, "Characterization of PEM fuel cell degradation by polarization change curves," J. Power Sources., 294, 82 (2015).

41. S. Torija, L. Prieto-Sanchez, and S. J. Ashton, "In-situ electrochemically active surface area evaluation of an open-cathode polymer electrolyte membrane fuel cell stack," J. Power Sources., 327, 543 (2016).

42. A. Pokhrel, M. El Hannach, F. P. Orfino, M. Dutta, and E. Kjeang, "Failure analysis of fuel cell electrodes using three-dimensional multi-length scale X-ray computed tomography," J. Power Sources., 329, 330 (2016).

43. T. Sasabe, P. Deevanhxay, S. Tsushima, and S. Hirai, "Soft X-ray visualization of the liquid water transport within the cracks of micro porous layer in PEMFC," Electrochem. Commun., 13, 638 (2011). 